\title{
Perspective
}

\section{MED12 mutations in human diseases}

\author{
Hua Wang ${ }^{1}$, Qin Shen ${ }^{2}$, Li-hua Ye ${ }^{1}$, Jun Ye ${ }^{1 \bowtie}$ \\ 1 Jiangsu Taizhou People's Hospital, Taizhou 225300, China \\ ${ }^{2}$ Taizhou Maternal and Children's Health-Care Center, Taizhou 225300, China \\ $\triangle$ Correspondence: yejun@gotofcm.com
}

The Mediator Complex plays key roles in activating gene transcription in eukaryotes. Mediator of RNA polymerase II transcription subunit 12 homolog (MED12) is a subunit of the Mediator Complex and regulates the activity of the complex. MED12 is involved in a variety of cellular activities, and mutations in MED12 gene impair MED12 activities and are associated with several diseases, including Opitz-Kaveggia syndrome, Lujan syndrome, uterine leiomyomas and prostate cancer. This review will discuss the biological function of MED12 and the relationship between MED12 mutations and diseases.

In eukaryotes, transcription of mRNAs requires two sequential steps. First, specific factors bind to DNA sequences and recruit co-factors to modify histone, which results in the relaxation of the bound DNA. Secondly, a multipleprotein complex named the Mediator Complex associates with the DNA and recruits transcription factors and RNA polymerase II (RNA Pol II) to initiate transcription (Malik and Roeder, 2010). In higher eukaryotes, the Mediator Complex is composed of at least 30 proteins (Bourbon, 2008), structurally divided into three core modules that are referred to as head, middle and tail. The head and middle modules interact directly with RNA Pol II, while the tail module associates with many co-factors to facilitate transcription. A kinase module interacts with the Mediator Complex to repress transcription (Knuesel et al., 2009a). The kinase module, composed of cyclin-dependent kinase 8 (CDK8), CycC, MED12 and MED13, binds to the core complex and thus interferes with the association of the Mediator Complex with RNA Pol II (Elmlund et al., 2006). MED12 is essential for assembly of the kinase module and thus affects the function of the Mediator Complex (Knuesel et al., 2009b). Here we review the biological functions of MED12, its involvement in diseases and the underlying mechanisms.

\section{THE BIOLOGICAL FUNCTIONS OF MED12}

MED12 is expressed in all eukaryotic species. In mammals, its gene is located on chromosome $\mathrm{X}$ (Kitano et al., 2003). MED12 consists of 2212 amino acids and is divided into four domains from the $\mathrm{N}$-terminus to $\mathrm{C}$-terminus: the leucine-rich domain $(L)$, the leucine/ serine-rich domain (LS), the proline/glutamine/leucine-rich domain (PQL) and the Opa domain.

As a component of the kinase module, MED12 can interact with G9a methyltransferase to induce histone methylation and thereby represses gene transcription (Ding et al., 2008). This function of MED12 is considered as an additional mechanism by which the kinase module negatively regulates the activity of the Mediator Complex.

MED12 interacts with different factors to regulate different biological activities. Transcriptional factor Nanog plays key roles in maintaining embryonic stem cells in the pluripotent state. It either activates or suppresses transcription depending on the promoters and differentiation states of cells. In embryonic stem cells, MED12 associates with $\mathrm{Na}$ nog to regulate gene expression (Tutter et al., 2009). MED12 also participates in the Wnt signaling pathway. When the pathway is activated, $\beta$-catenin is stabilized in the cytoplasm and translocated into the nucleus. It associates with MED12 to recruit the Mediator Complex to activate down-stream gene expression (Kim et al., 2006). In zebrafish neuronal development, MED12 is found to be important for the development of monoaminergic neurons and cranial sensory ganglia. MED12 regulates Sox9-mediated transcription activation of zash1a and lim1 (Wang et al., 2006). The function of MED12 is not confined to the nucleus. In the cytoplasm, MED12 associates with the immature form of TGF-bR2 to inhibit its glycosylation and thereby decreases TGF-bR2 levels at the plasma membrane (Huang et al., 2012). Based on these examples, one can tell that MED12 is involved in a variety of cellular activities. 


\section{THE RELATIONSHIP BETWEEN MED12 MUTATIONS AND HUMAN DISEASES}

Mutations in MED12 gene affect its biological functions. In humans, MED12 mutation may result in a variety of diseases, such as Opitz-Kaveggia syndrome, Lujan syndrome, uterine leiomyoma and prostate cancer. The relationship between MED12 mutations and human diseases and the underlying mechanisms are reviewed below.

\section{MED12 mutation and the X-linked mental retardation}

FG syndrome, also called Opitz-Kaveggia syndrome, was first reported by OpitzKaveggia in 1974 (Opitz and Kaveggia, 1974). It is an X chromosome-associated genetic disease. The main characteristics of the patients include short stature, mental retardation, anal atresia, congenital hypotonia, growth retardation in exercise capacity and part of corpus callosum dysplasia. Because FG syndrome shares common features with other diseases and lacks definitive markers for detection, it had been difficult to confirm the diagnosis for a long time. In 2007, Risheg found that there are frequent mutations in the twenty-first exon of MED12 of FG syndrome patients, which results in C2881T and R961W mutations in the protein. Thereafter, the relationship between FG syndrome and mutations in MED12 was confirmed by several other groups (Opitz and Kaveggia, 1974; Risheg et al., 2007; Graham et al., 2008; Graham et al., 2010). In addition, Rump P and colleagues found that three cousins suffering from FG syndrome all bear a mutation (G958E) in MED12, suggesting that the G958E mutation may also cause FG syndrome (Rump et al., 2011).

Lujan syndrome is a genetic neural growth retardation syndrome, which is characterized by being tall and thin, giant head, high and narrow face, maxillary dysplasia, hypotonia, mild to moderate mental retardation, abnormal behavior (such as hyperactivity, emotional instability, shyness, aggressiveness or autism) and dysplasia of corpus callosum. Although Lujan syndrome can be distinguished from Opitz-Kaveggia syndrome by some features (such as the height and head), there are many shared clinical characteristics for these two syndromes. It is thus difficult to accurately distinguish between the two diseases (Lacombe et al., 1993; Van Buggenhout and Fryns, 2006). Recent study found that Lujan syndrome is also associated with mutations in MED12. The mutations are in the twenty-second exon (A3020G; N1007S), adjacent to the mutation sites in the Opitz-Kaveggia syndrome (Schwartz et al., 2007).

How these mutations in MED12 cause mental retardation is not fully understood. Nonetheless, recent studies have provided some clues. Ding $\mathrm{N}$ et al. identified a protein interaction network involving MED12/Mediator, G9a methyltransferase and the RE1 silencing transcription factor (REST). MED12 bridges REST to G9a-dependent histone H3K9 dimethylation to suppress neuronal genes in non-neuronal cells. Mutations in MED12 found in FG and Lujan syndromes disrupt the REST repressor function, implicating that MED12associated mental retardation involves impaired REST-dependent neuronal gene regulation (Ding et al., 2008). Very recently, Lai et al. reported that MED12 interacts with a special class of activating long noncoding RNA (ncRNA-a) to regulate target gene expression. The Mediator complex containing mutant MED12 proteins found in the FG syndrome fails to interact with ncRNA-a. These results suggest that the loss of Mediator-ncRNA-a interactions may contribute to FG syndrome (Lai et al., 2013).

\section{MED12 mutations and uterine leiomyoma}

Uterine leiomyoma is a common benign tumor of the female reproductive system, caused by hyperplasia of the uterine smooth muscle and a small portion of connective tissue. Uterine leiomyoma displays a high incidence in reproductive age, and sixty-percent of the disease occurrs in women at around age 45 (Okolo, 2008; Selo-Ojeme et al.,
2008). Patients suffering from myoma of uterus usually have no symptoms. Severe patients may feel abdominal pain, menstrual disorder, hypermenorrhea and secondary anemia. Enlargement of myoma can cause urinary urgency, constipation and oppressive symptom. Myomatous anaplasia can also cause infertility (Stewart, 2001). Uterine leiomyoma can be attributed to many reasons, such as hormone disorders, early menopause and harmful genetic and psychological factors. However, the molecular mechanism for uterine leiomyoma is not clear yet. It has been reported that in patients suffering from uterine leiomyoma, MED12 mutations are found in stem cells, which leads to unlimited growth and tumor. The mutation sites in MED12 are located in the second exon (Makinen et al., 2011a, 2011b; Je et al., 2012; Markowski et al., 2012). It was recently reported that the second exon mutation occurs in $52.2 \%$ of uterine fibroid patients while the mutation is not found in other tumors (Je et al., 2012), implicating the involvement of MED12 in the disease.

Markowski et al. reported that mutations of MED12 are strongly associated with fibroids not displaying primary karyotypic alterations (Markowski et al., 2012). They also observed that significantly high levels of wingless-type MMTV integration site family member 4 (WNT4) is expressed in leiomyomas with MED12 mutations (Markowski et al., 2012). These results suggest that the MED12 mutation might be involved in the activation of the Wnt pathway that activates $\beta$-catenin, which is known to cause leiomyoma-like lesions in a mouse model. However, immunohistochemistry results showed that there is no correlation between MED12 status and $\beta$-catenin nuclear/cytoplasmic localization (Perot et al., 2012; Islam et al., 2013). Therefore, detailed mechanism for how MED12 mutations induce uterine leiomyoma awaits further investigation.

\section{MED12 mutations and uterine} malignant tumor

The relationship between benign uter- 
ine leiomyoma, leiomyosarcoma and malignant smooth muscle tumors with uncertain etiology (STUMP) is not clear yet (Hodge and Morton, 2007). It is controversially thought that uterine leiomyosarcoma is evolved from uterine leiomyoma. As described above, MED12 mutations exist in uterine leiomyoma. But whether MED12 mutations exist in uterine leiomyosarcoma and STUMP is not clear yet. Analyses of the status of MED12 in leiomyosarcoma and STUMP should help to address whether they develop from leiomyoma. Studies on a hot spot region of MED12 in thirty-three patients suffering uterine stromal tumor revealed that $66.6 \%$ of typical uterine leiomyomas patients had MED12 mutations, but only $11 \%$ of STUMP patients and $20 \%$ of uterine leiomyosarcoma patients had MED12 mutations (Perot et al., 2012). Some mutations in leiomyoma are also found in leiomyosarcoma, though the mutations seem rare in malignant tumors (Perot et al., 2012). Moreover, MED12 is expressed in all typical leiomyoma but in only $60 \%$ of atypical leiomyoma, $50 \%$ of STUMP and $20 \%$ of leiomyosarcoma (Perot et al., 2012). These results implicate that while not all leiomyosarcoma and STUMP develop from leiomyomas, a subset of leiomyoma has the potential for malignant progression.

\section{MED12 mutation and prostate cancer}

Prostate cancer is a kind of malignant tumor in prostate tissues, caused by prostate acinar cell's disorder and abnormal growth. The morbidity has geographic and racial differences. In Europe, United States and other developed countries, it is the second common malignant tumor in males (Daskivich et al., 2011; Jemal et al., 2011). In prostate cancer patients, the common gene changes include loss of NKX3.1 (8p21) and PTEN (10q23), increased androgen receptor (AR), and fusion of ETS family transcription factors genes with androgen promoter (He et al., 1997; Li et al., 1997; Nguyen and Wang, 2008). A recent study of 112 cases of prostate cancer revealed that MED12 mutations exist in prostate cancer patients but not in normal persons. Unlike uterine leiomyoma, the mutations of MED12 in prostatic carcinoma are found mainly in the epithelial cells but not in stromal stem cells. It has been proposed that MED12 mutations in prostate cancer may disrupt the androgen signaling pathway and the CDK8-dependent transcriptional regulation of p53 (Barbieri et al., 2012).

\section{SUMMARY AND PROSPECT}

Mediator Complex is a central regulator of transcription in eukaryotes. MED12 as a subunit of Mediator Complex plays a key role in transcriptional regulation. It regulates the growth and differentiation of cells, and participates in various signaling pathways. Mutations in MED12 cause such diseases as Lujan syndrome, Opitz-Kaveggia syndrome, uterine fibroids and prostate cancer. Investigation into the relationship between mutations and diseases is still in the beginning. It is predictable that more diseases will be found to be linked with MED12 mutations. Comparison of the gene expression profiles between patient and control samples should shed light on how MED12 mutations cause the diseases. Such investigation will provide new insights on mechanisms of the diseases and help to develop new therapy strategies.

\section{COMPLIANCE WITH ETHICS GUIDELINES}

Hua Wang, Qin Shen, Li-hua Ye and Jun Ye declare that they have no conflict of interest.

The article does not contain any studies with human or animal subjects performed by the any of the authors.

\section{REFERENCES}

Barbieri, C.E., Baca, S.C., Lawrence, M.S., Demichelis, F., Blattner, M., et al. (2012). Nat Genet 44, 685-689.

Bourbon, H.M. (2008). Nucleic Acids Res 36 , 3993-4008.

Daskivich, T.J., Chamie, K., Kwan, L., Labo, J., Palvolgyi, R., et al. (2011). Cancer 117, 2058-2066.

Ding, N., Zhou, H., Esteve, P.O., Chin, H.G.,
Kim, S., et al. (2008). Mol Cell 31, 347359.

Elmlund, H., Baraznenok, V., Lindahl, M., Samuelsen, C.O., Koeck, P.J., et al. (2006). Proc Natl Acad Sci U S A 103, 15788-15793.

Graham, J.M., Jr., Clark, R.D., Moeschler, J.B., and Rogers, R.C. (2010). Am J Med Genet C Semin Med Genet 154C, 477-485.

Graham, J.M., Jr., Visootsak, J., Dykens, E., Huddleston, L., Clark, R.D., et al. (2008). Am J Med Genet A 146A, 3011-3017.

He, W.W., Sciavolino, P.J., Wing, J., Augustus, M., Hudson, P., et al. (1997). Genomics 43, 69-77.

Hodge, J.C., and Morton, C.C. (2007). Hum Mol Genet 16 Spec No 1, R7-13.

Huang, S., Holzel, M., Knijnenburg, T., Schlicker, A., Roepman, P., et al. (2012). Cell 151, 937-950.

Islam, M.S., Protic, O., Stortoni, P., Grechi, G., Lamanna, P.,et al. (2013). Fertil Steril. pii: S0015-0282(13)00408-1.

Je, E.M., Kim, M.R., Min, K.O., Yoo, N.J., and Lee, S.H. (2012). Int J Cancer 131, E1044-1047.

Jemal, A., Bray, F., Center, M.M., Ferlay, J., Ward, E., et al. (2011). CA Cancer J Clin 61, 69-90.

Kim, S., Xu, X., Hecht, A., and Boyer, T.G. (2006). J Biol Chem 281, 14066-14075.

Kitano, T., Schwarz, C., Nickel, B., and Paabo, S. (2003). Mol Biol Evol 20, 1281-1289.

Knuesel, M.T., Meyer, K.D., Bernecky, C., and Taatjes, D.J. (2009a). Genes Dev 23, 439-451.

Knuesel, M.T., Meyer, K.D., Donner, A.J., Espinosa, J.M., and Taatjes, D.J. (2009b). Mol Cell Biol 29, 650-661.

Lacombe, D., Bonneau, D., Verloes, A., Couet, D., Koulischer, L., et al. (1993). Genet Couns 4, 193-198.

Lai, F., Orom, U.A., Cesaroni, M., Beringer, M., Taatjes, D.J., et al. (2013). Nature 494, 497-501.

Li, J., Yen, C., Liaw, D., Podsypanina, K., Bose, S., et al. (1997). Science 275, 1943-1947.

Makinen, N., Heinonen, H.R., Moore, S., Tomlinson, I.P., van der Spuy, Z.M., et al. (2011a). Oncotarget 2, 966-969.

Makinen, N., Mehine, M., Tolvanen, J., Kaasinen, E., Li, Y., et al. (2011b). Science 334, 252-255.

Malik, S., and Roeder, R.G. (2010). Nat Rev Genet 11, 761-772. 
Markowski, D.N., Bartnitzke, S., Loning, T., Drieschner, N., Helmke, B.M., et al. (2012). Int J Cancer 131, 1528-1536.

Nguyen, M.M., and Wang, Z. (2008). Minerva Urol Nefrol 60, 15-29.

Okolo, S. (2008). Best Pract Res Clin Obstet Gynaecol 22, 571-588.

Opitz, J.M., and Kaveggia, E.G. (1974). Z Kinderheilkd 117, 1-18.

Perot, G., Croce, S., Ribeiro, A., Lagarde, P., Velasco, V., et al. (2012). PLoS One 7, e40015.

Risheg, H., Graham, J.M., Jr., Clark, R.D., Rogers, R.C., Opitz, J.M., et al. (2007) Nat Genet 39, 451-453.

Rump, P., Niessen, R.C., Verbruggen, K.T., Brouwer, O.F., de Raad, M.,et al. (2011). Clin Genet 79, 183-188.

Schwartz, C.E., Tarpey, P.S., Lubs, H.A., Verloes, A., May, M.M., et al. (2007). J Med Genet 44, 472-477.

Selo-Ojeme, D., Lawal, O., Shah, J., Mandal,
R., Pathak, S., et al. (2008). J Obstet Gynaecol 28, 421-423.

Stewart, E.A. (2001). Lancet 357, 293-298.

Tutter, A.V., Kowalski, M.P., Baltus, G.A., lourgenko, V., Labow, M., et al. (2009). J Biol Chem 284, 3709-3718.

Van Buggenhout, G., and Fryns, J.P. (2006). Orphanet J Rare Dis 1, 26.

Wang, X., Yang, N., Uno, E., Roeder, R.G., and Guo, S. (2006). Proc Natl Acad Sci U S A 103, 17284-17289. 